\title{
NEU: recurso de aproximação do estudante de medicina com a comunidade
}

\author{
NEU as a resource connecting medicine students and the community
}

\begin{abstract}
Ana Clara Drummond Scarponi', Natália Fernanda Ferreira Brum², Gabriella Maciel Fiamoncini ${ }^{3}$, Angélica Fernandes Teixeira ${ }^{1}$, Bruna Pimenta Valente ${ }^{3}$, Karina Cristina Kopper ${ }^{4}$, Karina Vieira Resende Ignacchiti Pimentel ${ }^{3}$, Roberta Duarte Sampaio ${ }^{1}$, Sandra Carvalho de Almeida Braga ${ }^{3}$, Deborah Luíza Sicupira ${ }^{5}$, Fabiano Gonçalves Guimarães ${ }^{6}$
\end{abstract}

Scarponi ACD, Brum NFF, Fiamoncini GM, Teixeira AF, Valente BP, Kopper KC, Pimentel KVRI, Sampaio RD, Braga SCA, Sicupira DL, Guimarães FG. NEU: recurso de aproximação do estudante de medicina com a comunidade / NEU as a resource connecting medicine students and the community. Rev Med (São Paulo). 2017 jul.-set.;96(3):140-4.

RESUMO: Fazem parte da formação do profissional médico o fortalecimento da cidadania e a capacitação para atuar na promoção e prevenção da saúde em diferentes realidades socioculturais. Assim, o Núcleo de Extensão Universitária (NEU) do curso de medicina - UNIFENAS - BH foi planejado como um recurso que proporcionará o intercâmbio de saberes entre acadêmicos de medicina, docentes e a comunidade. Objetivo: o NEU propõe uma maior vinculação entre universitários e membros da comunidade, a fim de aproximá-los da realidade brasileira, bem como atuar em localidades carentes por meio de projetos variados, ressaltando a importância da medicina humanizada. Método: Em julho de 2016, o NEU, durante sete dias, realizou sua primeira expedição em Urucânia - MG. Foram desenvolvidas oficinas de música, higienização e abordados temas, como violência e educação sexual para crianças e adolescentes. Com a população adulta foi debatido sobre violência contra a mulher, vínculo afetivo, saúde do homem e amamentação. Por demanda da cidade, foi incluído um projeto para o centro de reabilitação de usuários de drogas.
Para o público idoso a abordagem incluiu oficinas de hipertensão e diabetes, grupo de caminhada e visitas ao asilo. Além do públicoalvo já mencionado, as atividades abrangeram educadores, agentes municipais de saúde e lideranças comunitárias. Resultados: As ações promovidas pelo NEU foram positivas para a população de Urucânia, bem como para os membros do grupo, possibilitando reflexões que engrandeceram a formação acadêmica e pessoal dos integrantes. Em relação às dificuldades, o grupo enfrentou, em alguns momentos, falta de estrutura, desinteresse de algumas instituições e a imprevisibilidade do público. Conclusão: Nessa expedição, a verdadeira essência do ser médico se mostrou efetiva, contribuindo para o desenvolvimento do município e para o fortalecimento do conceito de corresponsabilidade social entre acadêmico e comunidade.

Descritores: Relações comunidade-instituição; Assistência integral à Saúde; Participação da comunitária; Estudantes de medicina; Educação médica/recursos humanos; Humanização da assistência; Expedições.

Trabalho recebeu o prêmio Dr. João de Aguiar Pupo na categoria de Projetos Implantados/Consolidados no V Congresso SAMMG - Sociedade de Acadêmicos de Medicina de Minas Gerais, I CBMED - Congresso Brasileiro de Acadêmicos de Medicina, IV COBLAM - Congresso Brasileiro de Ligas Acadêmicas de Medicina, Belo Horizonte, 29 set. 1 out. 2016. Projeto apresentado como Comunicação Oral no 54 COBEM - Congresso Brasileiro de Educação Médica, Brasília, DF, 12-15 out. 2016.

1. Acadêmicos do $10^{\circ}$ período de medicina, Universidade José do Rosário Vellano, Belo Horizonte, MG, BR. E-mail: anaclara.scarponi@gmail.com, angelicafteixeira9@hotmail.com, beta.sampaio@hotmail.com.

2. Acadêmica do $8^{\circ}$ período de medicina, Universidade José do Rosário Vellano, Belo Horizonte, MG, BR. Email: nffbrum@gmail.com.

3. Acadêmicos do $11^{\circ}$ período de medicina, Universidade José do Rosário Vellano, Belo Horizonte, MG, BR. Email: gabifiamoncini@hotmail.com, bruna.p.valente@gmail.com, karina rip@hotmail.com, sandrinhacabraga@hotmail.com.

4. Acadêmica do $7^{\circ}$ período de medicina, Universidade José do Rosário Vellano, Belo Horizonte, MG, BR. Email: karinackopper@gmail.com.

5. Médica, Universidade José do Rosário Vellano, Belo Horizonte, MG, BR. Email: sicupira.deborah@hotmail.com.

6. Médico, coordenador do Projeto, Especialista em Medicina de Família e Comunidade, Universidade José do Rosário Vellano, Belo Horizonte, MG, BR. E-mail: fabiano.guimaraes@unifenas.br.

Endereco para correspondência: Ana Clara Drummond Scarponi. Rua Pedro Sigaud, 266, Apto 501. Bairro Grajaú, Belo Horizonte, MG, BR. CEP: 30431-160. E-mail: anaclara.scarponi@gmail.com. 
ABSTRACT: it is part of the training of the medical professional the strengthening of citizenship and the capacitation in health promotion and disease prevention in different sociocultural realities. Thus, the Extension Nucleus of Unifenas - BH (NEU) was planned as a resource that will provide an exchange of knowledge among medical academics, teachers and community. Objective: NEU proposes to bring medical students closer to the Brazilian reality, as well as to work in needy communities through varied projects. Method: In July of 2016, the NEU made its first expedition in Urucânia - MG, with a duration of seven days. For children and teenagers, music workshops were developed, sanitation in the APAE and topics such as violence and sex education were discussed. With the adult population other topics were discussed: violence against women, affective bond, human health and breastfeeding. Coming from the town's solicitation, a project for drug user rehabilitation center was developed. With the elderly population: hypertension and diabetes workshop, walking groups and asylum visits were conducted. The applied workshops

\section{INTRODUÇÃO}

az parte da graduação dos acadêmicos de
medicina a formação de profissionais-cidadãos capacitados para atuarem, direta e indiretamente, na promoção e prevenção da saúde em diferentes realidades sócio-culturais. Diante desse contexto a participação em projetos de extensão se torna uma ferramenta essencial para a consolidação do conhecimento técnico-científico, além de aproximar as instituições de ensino, como a Universidade José do Rosário Vellano - UNIFENAS, com as comunidades. Nesse sentido, foi desenvolvido o Núcleo de Extensão Universitária (NEU) do curso de Medicina - UNIFENAS - BH, planejado como um recurso que proporcionará a troca de saberes e experiências entre acadêmicos de medicina, docentes do curso e diversas comunidades dos municípios a serem selecionadas. O grupo surgiu em Janeiro de 2016, formado por dez acadêmicas e um Médico de Família e Comunidade que é professor do curso. No primeiro semestre de 2016 o projeto foi desenvolvido e legitimado pela UNIFENAS $\mathrm{BH}$, tendo como primeiro município de atuação a cidade de Urucânia - MG.

\section{OBJETIVO}

O NEU propõe uma maior vinculação entre o acadêmico de medicina e comunidade, a fim de aproximálos da realidade sociocultural brasileira, a partir do contato do estudante com a população local, políticas públicas e gestão local. Além disso, propiciar o desenvolvimento de projetos, criados por alunos, voltados para comunidades carentes; contribuir para a melhoria das condições de vida da população, por meio de ações coletivas nas áreas de educação, saúde, cultura e meio ambiente; bem como included several target groups: educators, municipal health agents and community leaders. Results: the actions promoted by the NEU were positive for the population of Urucânia, as well as for the members of the group. Thereafter, several reflections were generated that provided a set for an academic and personal formation of the members. In relation to the difficulties, the group faced, in some moments, lack of infrastructure; lack of interest in some institutions and unpredictability of the public. Conclusion: The actions carried out by NEU, in this expedition, contributed not only to the development of the town, but also to strengthen the concept of social responsibility between the academic program and community.

Keywords: Community-institutional relations; Comprehensive health care; Community participation; Students, medical; Education, medical/manpower; Humanization of assistance; Medicina comunitária/educação; Community, medicine/ education; Expeditions.

promover a capacitação de agentes multiplicadores para as atividades comunitárias. Tudo isso ressaltando a importância da humanização na medicina.

\section{DESCRIÇÃO}

A primeira expedição do NEU ocorreu em Urucânia - MG, município com 10.291 habitantes, segundo o senso de 2010 do Instituto Brasileiro de Geografia e Estatística (IBGE) e contou com a participação de dez alunos do curso de medicina da Unifenas - BH e um professor responsável Dr. Fabiano Guimarães, médico de família. A economia da cidade se sustenta principalmente pelo cultivo e beneficiamento da cana-de-açúcar e pela suinocultura. $\mathrm{O}$ local foi escolhido considerando a heterogeneidade sócioeconômica-cultural da região e a partir do contato com a prefeitura do município, que propôs intervenções que abrangessem diversos aspectos da população.

Após este primeiro contato com a gestão local, foi realizada uma viagem precursora para Urucânia, com a finalidade de identificar o público-alvo, de conhecer as principais demandas locais relacionadas à saúde, educação, cultura e aos aspectos sociais e de discutir a viabilidade de execução do projeto. Também foram apresentados à prefeitura dez projetos possíveis de serem executados na cidade e em alguns distritos, todos desenvolvidos pelas acadêmicas do grupo e orientados pelo professor responsável. As ações propostas pelos projetos apresentavam como público-alvo toda a população, além de educadores, servidores públicos, agentes municipais de saúde, lideranças comunitárias, representantes da sociedade civil, considerados possíveis multiplicadores. Para a realização das atividades foram consideradas estratégia seminários, grupos de discussões, oficinas e palestras. A divulgação das ações propostas para a cidade ficou a cargo da prefeitura. 
O terceiro momento da atuação do NEU foi a expedição, executada no período de uma semana - 03 a 10 de julho de 2016 onde acadêmicos e professor responsável se hospedaram no Centro de Saúde Paulo Giardinni. Foram desenvolvidas atividades voltadas para diversos públicos e os integrantes se dividiram em duplas, trios e em alguns momentos todos trabalharam em coletividade, propiciando assim, a realização de um maior número de atividades no mesmo dia. Os locais de atuação incluíram escolas, praças, centros de saúde, Centro de Referência em Assistência Social (CRAS), Associação de Pais e Amigos dos Excepcionais (APAE), asilo e centro de recuperação para usuários de drogas.

As ações desenvolvidas para crianças e adolescentes foram: abordagem sobre sexualidade e violência; aulas interativas com as crianças e professores da APAE, reforçando a importância da higienização. No CRAS foram elaboradas atividades de musicalidade com as crianças e os educadores como forma de estabelecer autoestima, auxiliar nos desafios da vida e afastá-las das drogas, violência e criminalidade, repassar valores culturais, desenvolver o interesse pela música e a criatividade; além de dinâmicas sobre vínculo afetivo entre pais e filhos com as mães das crianças.

Para o público adulto foram desenvolvidas: curso sobre humanização no atendimento para profissionais em saúde; palestra sobre violência contra a mulher para alunos da Educação de Jovens e Adultos (EJA), do magistério e população dos distritos mais afastados; palestras sobre os benefícios da amamentação, ministrada para gestantes e profissionais de saúde; encontros com professores das escolas municipais para discussão da violência sexual infantil; encontros com a população masculina para abordagem de vários aspectos da saúde do homem; ida ao centro de recuperação de usuários de drogas promovendo dinâmicas que estimulam a autoconfiança.

Com o público idoso: visitas ao asilo com o intuito de promover maior interação entre os idosos hospedados, por meio de dinâmicas, de música, palhaçaria; encontros com o grupo de caminhada da cidade, em que se discutiu a prática de atividade física segura, seus benefícios e exercícios de alongamento. Também foram realizadas reuniões com grupos operacionais para abordagem do tratamento, principalmente não medicamentoso, do diabetes e da hipertensão arterial sistêmica.

Além disso, o NEU desenvolveu dias de saúde na praça com orientações a respeito das mudanças no estilo de vida, aferição da pressão arterial, medição da glicemia capilar e foram realizadas visitas domiciliares acompanhadas com os Agentes Comunitários em Saúde (ACS) em áreas mais afastadas.

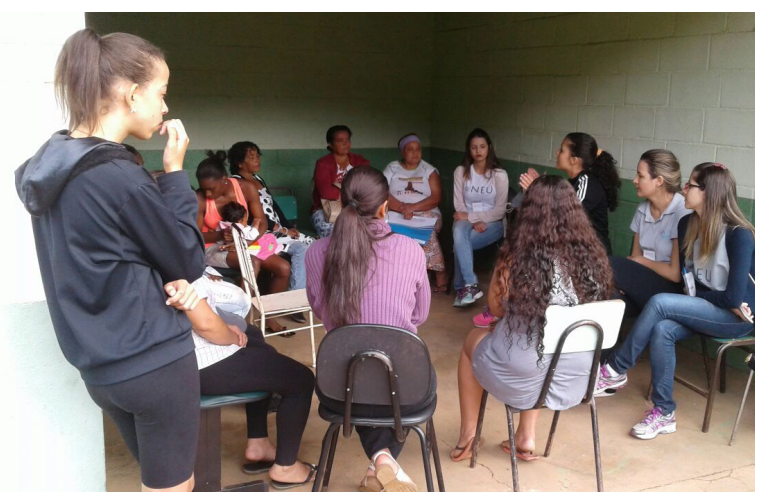

Figura 1 - Palestra, no Centro de Saúde de Jatiboca, sobre violência contra a mulher

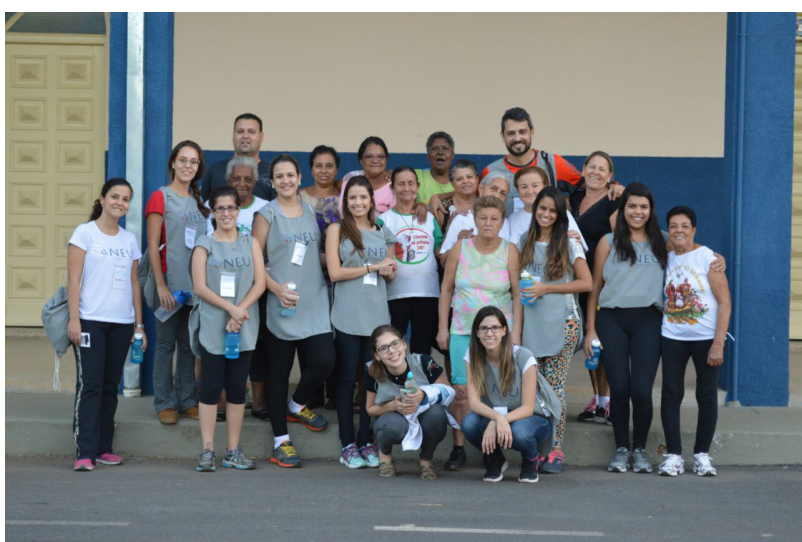

Figura 2 - Prática de atividades físicas com o grupo de caminhada da cidade de Urucânia

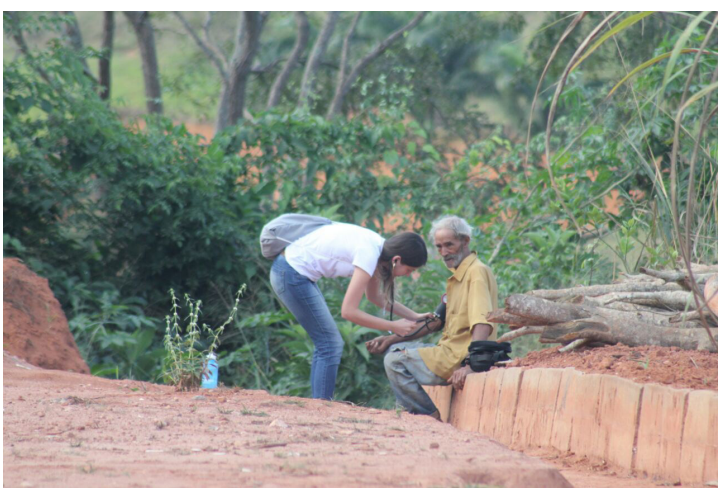

Figura 3 - Visita ao asilo de Urucânia

\section{RESULTADOS}

Parcelas diversas da população participaram, ativamente, das atividades desenvolvidas pelo NEU, Boa parte dessas ações não foram divulgadas com antecedência à comunidade, como acertado previamente em reunião com a prefeitura da cidade, comprometendo algumas atividades e exigindo maior empenho do grupo.

Os projetos realizados com o público infantojuvenil foram bem-sucedidos, com grande adesão deste público às atividades. Durante as palestras sobre 
educação sexual abordou-se sobre mudanças corporais, físicas e psicológicas, métodos contraceptivos, doenças sexualmente transmissíveis e violência sexual. Com relação aos usuários da APAE, foi solicitado, pela direção do local, tratar com o público alvo crianças, jovens e docentes sobre a importância da higienização corporal, com destaque para saúde bucal e assepsia das mãos. Durante as atividades musicais, as crianças puderam refletir sobre a paz e a felicidade por meio de músicas, trabalharam a coordenação motora e ensaiaram algumas canções que foram apresentadas, em forma de coral, no encerramento da expedição do NEU. Esta ação foi capaz de promover a união das crianças com seus responsáveis e despertou o interesse dos funcionários do CRAS como um projeto a ser continuado na instituição. As atividades sobre vínculo afetivo foram trabalhadas de forma lúdica entre pais e filhos, a partir de dinâmicas, brincadeiras e palestras, com o intuito de promover uma maior união familiar, prevenindo assim, o envolvimento dos filhos com a violência e proporcionando uma maior aproximação entre os membros da família.

As oficinas realizadas com os adultos atingiram um número elevado de participantes e cada membro do grupo foi responsável por uma ação. O curso sobre Humanização no atendimento em Saúde contou com uma diversidade de profissionais da atenção primária e da gestão da cidade, porém, os participantes demonstraramse dispersos e desinteressados, foram abordadas diversas dificuldades enfrentadas pelos profissionais da saúde, sendo esclarecidas algumas condutas para lidar com as diversas problemáticas debatidas. A qualidade das condições de trabalho, inclusão dos sujeitos na tomada de decisões nos serviços de saúde, precariedade no atendimento recebido e a não garantia de atendimento longitudinal nos níveis de atenção foram algumas pautas do curso. As palestras sobre violência contra a mulher, ministrada aos alunos do EJA, da magistratura e para a população dos distritos mais próximos da cidade foram capazes de abordar: histórico de violência contra a mulher, formas de denúncia, defesa das vítimas, tipos de violência, aspectos psicossociais, etc. A saúde materna foi debatida na forma de encontros com as gestantes, com foco no aleitamento materno. Nestes grupos discutiramse as vantagens do aleitamento materno e vínculo afetivo entre mãe e filho. Esta foi uma ótima oportunidade para o esclarecimento de dúvidas, mitos e direitos trabalhistas. A aula se dividiu em parte teórica e prática e a presença de profissionais foram fundamentais para a continuidade do grupo de gestantes. A última atividade realizada com os adultos buscou esclarecer vários aspectos da saúde do homem, como a andropausa, disfunção erétil, hiperplasia prostática benigna e câncer de próstata. Os ouvintes mostraram-se interessados e dúvidas foram escutadas no decorrer destas reuniões.

$\mathrm{O}$ encontro com os professores sofreu algumas modificações durante a expedição. Inicialmente, a ideia do grupo era abordar com os professores e direção como conduzir casos de suspeita/confirmação de abuso sexual infanto-juvenil, entretanto, a diretoria da escola não permitiu a realização desta atividade e de outras, como abuso de álcool e drogas, com a justificativa de não serem temáticas enriquecedoras para a instituição em questão. Após o NEU se reunir e discutir esta problemática, foi percebido, o receio das escolas em confrontar e assumir realidades como estas. O tema pôde ser discutido em apenas uma escola da cidade, porém com pouca adesão dos professores à atividade.

As oficinas e palestras voltadas para o público idoso foram realizadas durante reunião do grupo dos idosos, contando com a participação de um grande número de ouvintes. Este público alvo é bem assistido no município, que conta com atividades específicas que proporcionam uma melhor qualidade de vida. Além disso, foram realizadas visitas ao asilo. Este, Apesar de ter uma infraestrutura adequada para a hospedagem dos usuários, uma demanda solicitada ao NEU foi a melhora do convívio e a interação entre os usuários. Com este objetivo, foram elaboradas dinâmicas e atividades que buscaram aproximar o vínculo afetivo entre os internos. $\mathrm{O}$ grupo promoveu atividades recreativas, com música, dança e brincadeiras. A resposta foi imediata e muitos idosos elogiaram as intervenções realizadas. Nos encontros com o grupo de caminhada da cidade foi proposto a realização de exercícios de alongamentos respeitando as limitações dos participantes, instruindo sobre os benefícios desta atividade e dicas de como se cuidar para exercer uma prática esportiva segura.

Em relação aos grupos operativos de diabetes e hipertensão, a ênfase do debate foram as mudanças no estilo de vida. O grupo discutiu com a população e os profissionais de saúde sobre o local adequado para o armazenamento de insulina, combinações de alimentos para uma refeição balanceada, riscos das complicações frente ao mau controle glicêmico e pressórico e esclarecimento de dúvidas e mitos associados a estas doenças crônicas.

\section{CONCLUSÃO}

As ações realizadas pelo NEU na expedição Urucânia contribuíram para o desenvolvimento do município, e também para fortalecer do conceito de cidadania entre acadêmico e comunidade.

A região possui problemas de relevância social e a partir deste contexto o NEU buscou atuar de forma a contribuir para o desenvolvimento sustentável da 
Scarponi ACD, et al. NEU: recurso de aproximação do estudante de medicina com a comunidade.

comunidade, por meio principalmente da capacitação de agentes multiplicadores e da instrução da população.

Experiência extensionista como esta é capaz de promover reflexões a todos os estudantes que nelas se envolvem, pois, a percepção sobre a responsabilidade social e a crítica sobre o comportamento do indivíduo enquanto cidadão são marcantes no decorrer da expedição. Com isso, um projeto quando sai do papel e se torna ação, escolhe ser comunidade, e assim, vive transformando vidas e promovendo a essência da extensão, atravessando os muros da faculdade e adentrando na verdadeira humanização.

artigo recebido em:16.05.2017

Artigo aceito em: 06.06.2017 